\title{
In Reply: Reliability of Bony Landmarks to Predict Intradural Location of Paraclinoid Aneurysms
}

\author{
Etienne Lefevre ${ }^{1,2}$ (D) - Caroline Apra ${ }^{1} \cdot$ Salomao Faroj Chodraui-Filho ${ }^{2}$ - Dorian Chauvet ${ }^{1} \cdot$ Stanislas Smajda $^{2}$. \\ Michel Piotin ${ }^{2} \cdot$ Robert Fahed ${ }^{2}$
}

Received: 27 July 2020 / Accepted: 14 September 2020 / Published online: 7 October 2020

(c) Springer-Verlag GmbH Germany, part of Springer Nature 2020

We would like to thank the authors for their thoughtful comments regarding our recent publication $[1,2]$. We are grateful for the opportunity to discuss issues pertaining to the interpretation of our observations, especially regarding the bony landmarks used for our study.

We have read their last paper with great interest and fully agree with the authors regarding the accuracy of computed tomography angiography (CTA) for approaching the exact location of the distal dural ring (DDR) [3]. This article is by the way the one we used to create a reading protocol for our study; however, flourishing literature about the existence of the carotid cave (CC), an intradural pouch that extends below the level of the DDR, might question the utility of locating the DDR as it is not considered to be the anatomical limit of intradural space anymore $[4,5]$.

Concerning the optic strut (OS) line, there was indeed only one OS line in each patient, delimited by connecting the two points at bilateral bases of the OS. Connecting these two points to form a line was a way of standardizing the axial angle between raters in order for this evaluation to be reproducible.

Concerning Fig. 2c, the OS line was defined on the coronal view that showed both OS; however, the aneurysm was not always visible on the coronal view that allowed the detection of the OS, even when maximum intensity projection (MIP) was used, and the readers sometimes had to scroll through the images to then locate the aneurysm. This is the

The manuscript has not been submitted elsewhere nor published elsewhere in whole or in part.

Etienne Lefevre

etienne.lefevre@neurochirurgie.fr

1 Neurosurgery Department, Rothschild Foundation Hospital, 75019 Paris, France

2 Interventional Neuroradiology Department, Rothschild Foundation Hospital, 75019 Paris, France reason why the OS line does not seem to be in contact with the OS on Fig. 2c.

Anatomically, paraclinoid aneurysms can be classified as intradural, extradural or transitional when their neck or dome is located above, below or across the DDR, respectively; however, our main objective in daily practice is to assess the risk of subarachnoid hemorrhage (SAH) of these aneurysms. Therefore, the aim of this study was to evaluate the ability of the described bony landmarks to clinically classify these aneurysms as "at risk of SAH" or "not at risk of SAH". In our series, all the patients had a SAH, therefore even the aneurysms classified as "transitional" by the readers are thought to carry a high probability of being responsible for the $\mathrm{SAH}$.

We are delighted that the present paper creates such interest and we hope that our response satisfies the authors and other readers with the same interrogations.

Conflict of interest E. Lefevre, C. Apra, S. F. Chodraui-Filho, D. Chauvet, S. Smajda, M. Piotin and R. Fahed declare that they have no competing interests.

\section{References}

1. Lefevre E, Apra C, Chodraui-Filho SF, Chauvet D, Smajda S, Piotin M, Fahed R. Reliability of bony landmarks to predict intradural location of paraclinoid aneurysms. Clin Neuroradiol. 2020. https:// doi.org/10.1007/s00062-020-00896-0.

2. Hsu SP, Lin CF, Liao CH. Optic strut and tuberculum sellae as reliable radiological landmarks to predict intradural location of paraclinoid aneurysms in CT angiography. Clin Neuroradiol. 2020. https:// doi.org/10.1007/s00062-020-00917-y.

3. Liao CH, Lin CJ, Lin CF, Huang HY, Chen MH, Hsu SP, Shih YH Comparison of the effectiveness of using the optic strut and tuberculum sellae as radiological landmarks in diagnosing paraclinoid aneurysms with CT angiography. J Neurosurg. 2016;125:275-82.

4. Kobayashi S, Kyoshima K, Gibo H, Hegde SA, Takemae T, Sugita K. Carotid cave aneurysms of the internal carotid artery. J Neurosurg. 1989;70:216-21.

5. Joo W, Funaki T, Yoshioka F, Rhoton AL Jr. Microsurgical anatomy of the carotid cave. Neurosurgery. 2012;70(2 Suppl Operative):300-11. discussion 311-2. 